\title{
Prognostic Significance of Systemic Inflammatory Response in Patients with Synchronous and Metachronous Metastatic Renal Cell Carcinoma Receiving First-Line Tyrosine Kinase Inhibitors
}

\author{
Joongwon Choi ${ }^{1}$, Tae Jin Kim', Hyun Hwan Sung ${ }^{1}$, Hwang Gyun Jeon', \\ Byong Chang Jeong ${ }^{1}$, Seong Soo Jeon ${ }^{1}$, Hyun Moo Lee ${ }^{1}$, \\ Han Yong Choi ${ }^{2}$, Minyong Kang ${ }^{1}$, Seong II Seo \\ ${ }^{1}$ Department of Urology, Samsung Medical Center, Sungkyunkwan University School of Medicine, Seoul, Korea \\ ${ }^{2}$ Department of Urology, Kangbuk Samsung Hospital, Seoul, Korea
}

\begin{abstract}
Purpose: To determine whether systemic inflammatory response (SIR), particularly platelet-lymphocyte ratio (PLR) and neutrophil-lymphocyte ratio (NLR), has different prognostic role between patients with metastatic renal cell carcinoma (mRCC) receiving first-line tyrosine kinase inhibitors (TKI).

Materials and Methods: We retrospectively reviewed 547 patients with $\mathrm{mRCC}$ who were diagnosed and treated with a first-line TKI between 2007 and 2015. The primary endpoint was overall survival (OS) and secondary endpoint was progression-free survival (PFS). We evaluated differences in survival outcomes according to SIR and identified predictors of OS and PFS.

Results: In synchronous mRCC, patients with a higher PLR had significantly worse OS and PFS. Moreover, a higher NLR was also associated with both worse OS and PFS in these patients. However, PLR was not associated with either OS or PFS in metachronous mRCC patients. While metachronous mRCC patients with a higher NLR had worse OS compared to those with lower NLR, there was no difference in PFS according to the status of NLR. On multivariate analysis, PLR was identified as predictive factor for OS (hazard ratio [HR], 1.55) as well as PFS (HR, 1.39) in patients with synchronous $\mathrm{mRCC}$, but not in patients with metachronous mRCC. Additionally, higher NLR was also remained as predictive factor of both OS (HR, 1.83) and PFS (HR, 1.57) in patients with synchronous mRCC.

Conclusions: Our study indicates that simple biomarkers of SIR, particularly PLR and NLR, can be more useful predictors of survival outcomes in patients with synchronous mRCC rather than metachronous mRCC. (Korean J Urol Oncol 2019;17:150-159)
\end{abstract}

Key Words: Metastatic renal cell carcinoma $\cdot$ Metachronous $\cdot$ Prognosticator $\cdot$ Synchronous $\cdot$ Systemic inflammatory response $\cdot$ Tyrosine kinase inhibitor

Received September 9, 2019, Revised October 1, 2019, Accepted October 17, 2019

Comesponding Author: Seong Il Seo

Department of Urology, Samsung Medical Center, Sungkyunkwan University School of Medicine, 81 Irwon-ro, Gangnam-gu, Seoul 06351, Korea E-mail: seongil.seo@samsung.com, Tel: +82-2-3410-3556, Fax: +82-2-3410-6992

ORCID: https://orcid.org/0000-0002-9792-7798

Co-corresponding Author: Minyong Kang

Department of Urology, Samsung Medical Center, Sungkyunkwan University School of Medicine, 81 Irwon-ro, Gangnam-gu, Seoul 06351, Korea E-mail: dr.minyong.kang@gmail.com, Tel: +82-2-3410-1138, Fax: +82-2-3410-6992

ORCID: https://orcid.org/0000-0002-6966-8813

- This study was funded by a research grant from the National Research Foundation (NRF) of Korea, funded by the Ministry of Science and ICT (2018M3A9H1078336). This research was also supported by a grant from the Korea Health Technology R\&D Project through the Korea Health Industry Development Institute (KHIDI), funded by the Ministry of Health \& Welfare, Republic of Korea (HI17C0025).

(i) (7) This is an Open Access article distributed under the terms of the Creative Commons Attribution Non-Commercial License (http://creativecommons.org/licenses/by-nc/4.0/) which permits unrestricted non-commercial use, distribution, and reproduction in any medium, provided the original work is properly cited.

2019 (C) Copyright The Korean Urological Oncology Society and The Korean Prostate Society. All Rights Reserved. 


\section{INTRODUCTION}

The treatment of metastatic renal cell carcinoma (mRCC) has evolved continuously over the last 2 decades as evidenced by an increase in the 5-year survival rate of advanced $\mathrm{mRCC}$ over time (from $7.3 \%$ to $12.3 \%$ ). ${ }^{1}$ Targeted therapy with tyrosine kinase inhibitor (TKI) has been the standard of systemic treatment for patients with $\mathrm{mRCC}$ for more than 10 years. In mRCC patients with clear cell histology, sunitinib or pazopanib is recommended as first-line therapy after tissue sampling. Otherwise, sunitinib, cabozantinib or everolimus is recommended in nonclear cell histology patients.

Recently, immunotherapy by using immune checkpoint inhibitor has been received a great interest as a novel drug for mRCC. Nivolumab, a programmed death 1 checkpoint inhibitor, has proved that it is more effective than everolimus with advanced RCC patients in CheckMate 025 study. ${ }^{2}$ Furthermore, in CheckMate 214 study, nivolumab plus ipilimumab have significantly higher overall survival and objective response rates than sunitinib in same patient population. ${ }^{3}$ The effects of many immuno-oncology agent have been demonstrated, ${ }^{4,5}$ and it is apparent that these agents leading a paradigm shift in systemic therapy for $\mathrm{mRCC}$.

As such, the treatment of mRCC continues to develop, optimal treatment for these patients is still obstacle due to the lack of useful biomarker. Although treatment response assessment has been calculated by abdominal and chest computed tomography (CT) or bone scan, the evaluation method for indeterminate lesion is not yet known.

Blood markers of systemic inflammatory response (SIR) such as platelet-lymphocyte ratio (PLR) and neutrophil-lymphocyte radio (NLR) are suggested as prognostic factors in patients with various type of malignancy. ${ }^{6-8}$ Among many SIR markers, an elevated NLR is a strong indicator of poor prognosis in patients with prostate cancer, bladder cancer, and RCC. ${ }^{9-12}$ Also, an elevated PLR is associated with poor oncological outcomes in cancer patients. ${ }^{13,14}$ Considering the emerging role of immune-oncologic drugs in mRCC, SIR can be a simple and valuable biomarker.

Metastatic RCC is classified as either synchronous or metachronous. However, according to our knowledge, there is no report on different prognostic role of SIR between these 2 types of mRCC. Here, we aimed to determine whether PLR and NLR are prognostic factors of survival outcomes in mRCC who received first-line TKI. In particular, we focused on the different prognostic roles of these biomarkers between synchronous and metachronous mRCC patients.

\section{MATERIALS AND METHODS}

\section{Study Population}

We retrospectively reviewed the clinical data of 547 patients with mRCC. Among these patients, 59 patients with no values for NLR and PLR before treatment and 11 patients using the mTOR inhibitors were excluded from the study. We finally analyzed 261 synchronous and 216 metachronous mRCC patients who were treated with first-line systemic TKI between 2007 and 2015. The Institutional Review Board of Samsung Medical Center approved this study (IRB No. SMC 2019-01-165).

\section{Study Design}

We reviewed the presence of distant metastasis at the time of RCC diagnosis to classify synchronous and metachronous mRCC. Synchronous mRCC refers to metastatic disease present at the time of RCC diagnosis. Metachronous mRCC refers to cases where a nephrectomy is performed for primary RCC removal, and metastatic disease is identified on follow-up study. ${ }^{15}$

We reviewed various clinical factors, including age at time of metastasis-diagnosis, age at the time of treatment initiation, sex, the presence of hypertension and diabetes mellitus, body mass index $\left(\mathrm{kg} / \mathrm{m}^{2}\right)$, number of metastatic sites, pathologic $\mathrm{T}$ stage, Fuhrman nuclear grade, type of first-line TKI, and the International Metastatic Renal Cell Carcinoma Database criteria for $\mathrm{mRCC}$ prognosis. All patients had full image staging by CT of the chest, abdomen, and pelvis. The pathological staging and subtype of RCC samples were determined using the American Joint Committee on Cancer TNM system and the Heidelberg recommendations.

Total blood sampling was performed for hematological testing prior to TKI treatment. NLR was calculated as the 
ratio of absolute neutrophil count to absolute lymphocyte count measured in peripheral blood. PLR was measured in the same manner as the ratio of platelet count to absolute lymphocyte count. Cutoff values of PLR and NLR were divided based on the median value of the population. Pretreatment PLR and NLR cutoff values were 110.5 and 1.9 , respectively, for synchronous mRCC. With respect to metachronous mRCC, the PLR and NLR cutoff values were

Table 1. Baseline clinical characteristics

\begin{tabular}{|c|c|c|c|}
\hline Characteristic & Synchronous $(n=261)$ & Metachronous $(\mathrm{n}=216)$ & p-value \\
\hline mRCC-diagnosed age (yr) & $57.0(50.0-65.0)$ & $60.0(53.0-68.0)$ & 0.060 \\
\hline mRCC-treatment age (yr) & $57.0(50.0-66.0)$ & $61.0(54.0-69.0)$ & 0.021 \\
\hline Sex & & & 0.910 \\
\hline Male & $205(78.5)$ & $170(79.4)$ & \\
\hline Female & $55(21.2)$ & $44(20.6)$ & \\
\hline Hypertension & $106(41.2)$ & $95(44.6)$ & 0.512 \\
\hline Diabetes mellitus & $39(14.9)$ & $47(21.8)$ & 0.057 \\
\hline Body mass index $\left(\mathrm{kg} / \mathrm{m}^{2}\right)$ & & & 0.155 \\
\hline$>25$ & $199(78.3)$ & $148(72.2)$ & \\
\hline$\leq 25$ & $55(21.7)$ & $57(27.8)$ & \\
\hline Clinical $\mathrm{T}$ stage & & & $<0.001$ \\
\hline$\leq \mathrm{cT} 2$ & $104(39.8)$ & $121(56.0)$ & \\
\hline$\geq \mathrm{cT} 3$ & $142(54.4)$ & $64(29.6)$ & \\
\hline Surgery type & & & $<0.001$ \\
\hline No or unknown & $89(34.1)$ & $26(12.0)$ & \\
\hline Radical nephrectomy & $168(64.4)$ & $177(81.9)$ & \\
\hline Partial nephrectomy & $4(1.5)$ & $13(6.0)$ & \\
\hline \multicolumn{4}{|l|}{ Pathology status } \\
\hline Pathologic $\mathrm{T}$ stage & & & $<0.001$ \\
\hline $\mathrm{T} 1$ & $28(15.1)$ & $91(42.9)$ & \\
\hline $\mathrm{T} 2$ & $24(13.0)$ & $45(21.2)$ & \\
\hline $\mathrm{T} 3$ & $121(65.4)$ & $76(35.8)$ & \\
\hline $\mathrm{T} 4$ & $12(6.5)$ & $0(0)$ & \\
\hline Histology & & & 0.333 \\
\hline Clear cell & $165(92.2)$ & $196(89.5)$ & \\
\hline Papillary & $13(7.3)$ & $17(7.8)$ & \\
\hline Chromophobe & $1(0.6)$ & $6(2.7)$ & \\
\hline Fuhrman grade & & & $<0.001$ \\
\hline Low & $11(6.0)$ & $55(26.4)$ & \\
\hline High & $172(94.0)$ & $153(73.6)$ & \\
\hline No. of metastasis & & & 0.053 \\
\hline Single & $106(40.6)$ & $107(49.5)$ & \\
\hline Multiple & $155(59.4)$ & $109(50.5)$ & \\
\hline Tyrosine kinase inhibitor types & & & 0.472 \\
\hline Sunitinib & $149(57.3)$ & $129(60.3)$ & \\
\hline Sorafenib & $31(11.9)$ & $25(11.7)$ & \\
\hline Pazopanib & $73(28.1)$ & $56(26.2)$ & \\
\hline \multicolumn{4}{|l|}{ Complete blood counts } \\
\hline $\mathrm{WBC}(/ \mu \mathrm{L})$ & $5,590(4,295-7,680)$ & $5,740(4,353-7,438)$ & 0.805 \\
\hline Hemoglobin (g/dL) & $12.6(10.8-13.9)$ & $13.4(11.9-14.8)$ & $<0.001$ \\
\hline Platelet $\left(\times 10^{3} / \mu \mathrm{L}\right)$ & $183.0(126.0-261.5)$ & $175.0(122.5-231.3)$ & 0.143 \\
\hline Neutrophil $(/ \mu \mathrm{L})$ & $2,950(1,940-4,735)$ & $3,036(1,875-4,260)$ & 0.466 \\
\hline Lymphocyte $(/ \mu \mathrm{L})$ & $1,570(1,101-2,201)$ & $1,889(1,437-2,400)$ & $<0.001$ \\
\hline
\end{tabular}

Values are presented as median (interquartile range) or number (\%). mRCC: metastatic renal cell carcinoma, WBC: white blood cell. 
90.4 and 1.5 , respectively.

\section{Statistical Analysis}

The primary endpoint of the current study was overall survival (OS), defined as the time from initiation of targeted therapy to time of death. Progression-free survival (PFS) was used as a secondary endpoint, and it was defined as time between initiation of targeted therapy to progression, cessation of treatment, or death. Kaplan-Meier curve analysis and log-rank tests were used to compare survival outcomes between groups. Multivariate Cox regression hazard ratio model was used to identify the predictive factors of OS. All descriptive data are presented as the median with interquartile range or percentage (\%) of events. Student t-test was used to compare continuous variables and the chi-square test was used to compare categorical variables. Data were analyzed with IBM SPSS Statistics ver. 23.0 (IBM Co., Armonk, NY, USA) and GraphPad Prism software (GraphPad Software Inc., San Diego, CA, USA). All

A
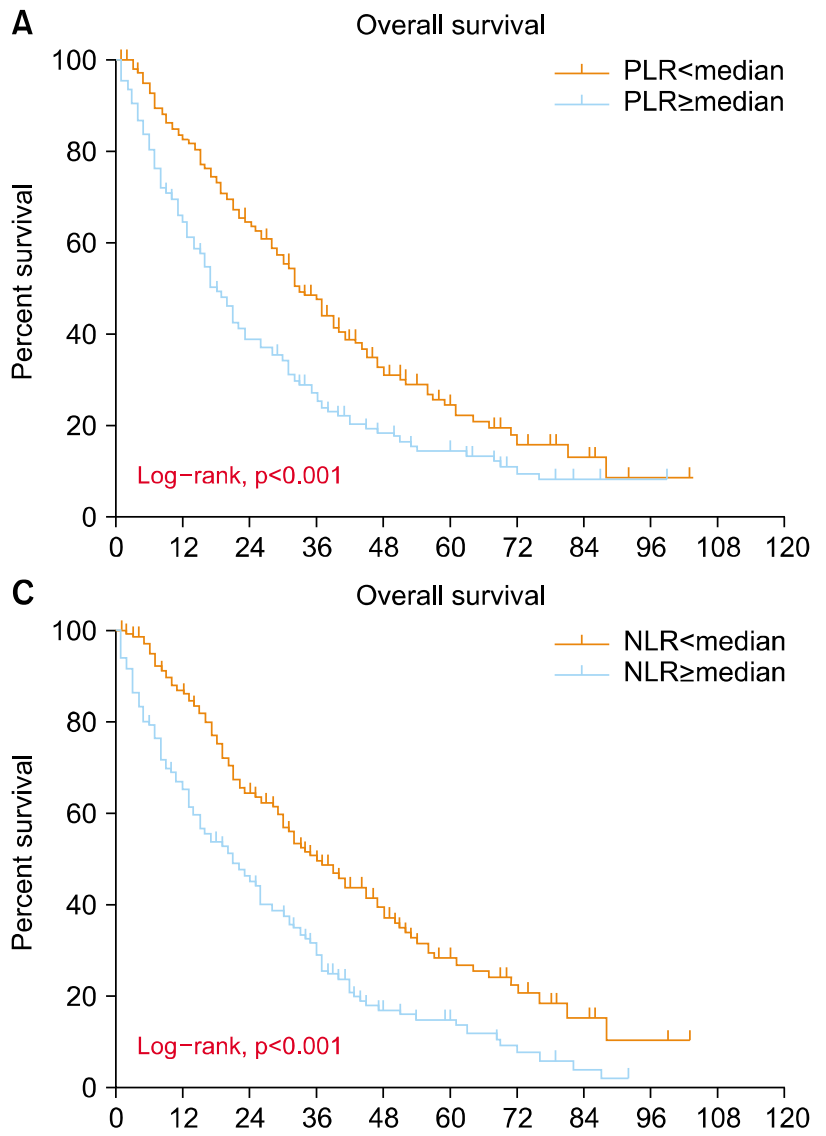

statistical tests were 2 sided, and statistical significance was defined as $\mathrm{p}<0.05$.

\section{RESULTS}

Baseline demographics are presented in Table 1. Patient with synchronous mRCC tended to be younger at the time of TKI treatment. With respect to pathological status, there was no difference in the histology of the 2 groups. The most common pathological $\mathrm{T}$ stage was T3 (65.4\%) in the synchronous population and T1 (42.9\%) in the metachronous population. There were more cases of Fuhrman high-grade disease in the synchronous population compared to the metachronous population $(94.0 \%$ vs. $73.6 \%$, respectively).

In the overall $\mathrm{mRCC}$ population, patients with a higher PLR exhibited poorer OS and PFS outcomes compared to those with a lower PLR (Fig. 1). Likewise, a higher NLR was associated with worse OS and PFS in the overall

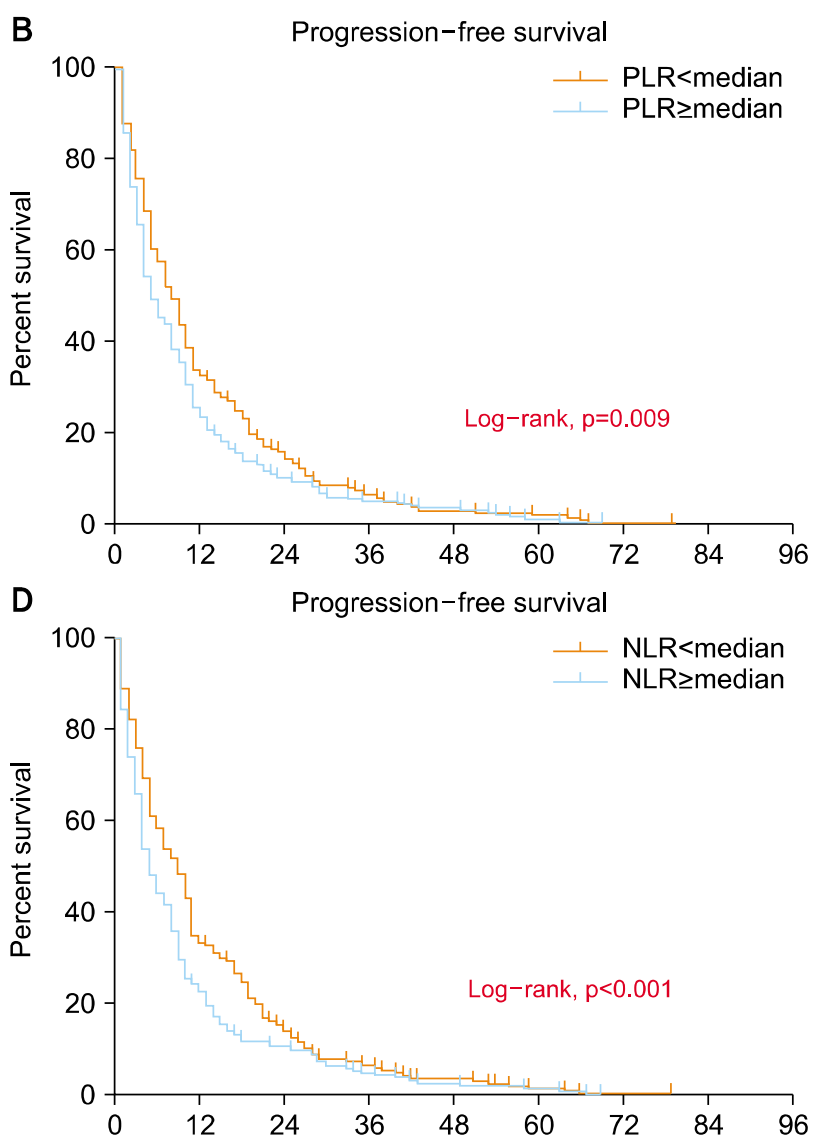

Fig. 1. Overall survival and progression-free survival in overall metastatic renal cell carcinoma patients stratified by median platelet-lymphocyte ratio (PLR) (A, B) and neutrophil-lymphocyte ratio (NLR) (C, D). 
mRCC population. Interestingly, patients with a higher PLR showed significantly worse and PFS outcomes than those with a lower PLR in the synchronous mRCC population (Fig. 2). In these population, a higher NLR also associated with worse OS and PFS rates. Conversely, there were no differences in terms of OS and PFS outcomes according to the status of PLR in the metachronous $\mathrm{mRCC}$ population (Fig. 3). Additionally, there was no difference in PFS according to the status of NLR. A higher NLR was only associated with poorer OS outcomes in patients with metachronous mRCC.

In multivariate Cox regression analysis, NLR and PLR are verified as the predicator of OS (Table 2, Supplementary Table 1) and the PFS (Supplementary Tables 2, 3) in synchronous mRCC population. But in metachronous population, PLR was not act as a predicator in neither OS (Table 3) nor PFS (Supplementary Table 4). NLR wasn't predicator in PFS (Supplementary Table 5), but remained as predictive factor of OS (Supplementary Table 6).

A
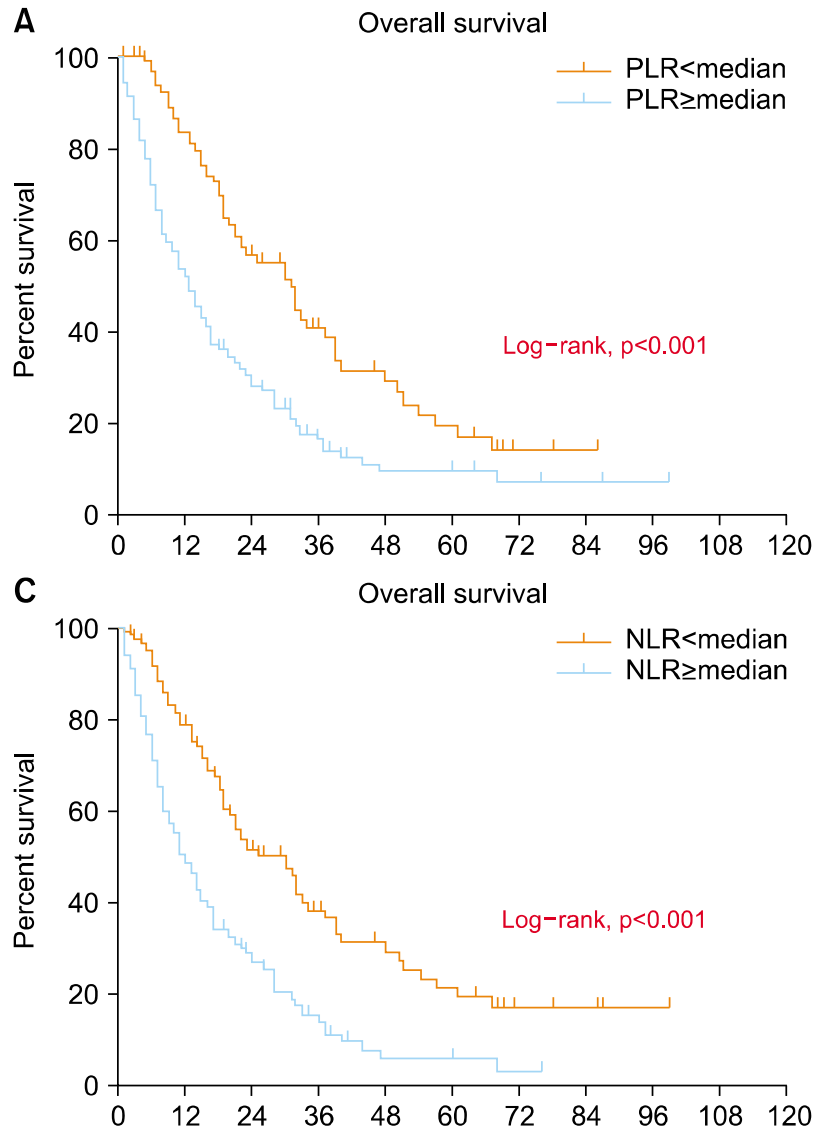

\section{DISCUSSION}

Szendi et al. ${ }^{16}$ made the initial report of synchronous and metachronous RCC metastasis in 1959, and since then many studies have been conducted to compare these 2 forms of disease. The biology and outcomes of synchronous and metachronous mRCC are different. ${ }^{17}$ Considering that SIR is a prognostic factor that reflects interactions between tumor and host, it is reasonable to expect that the role of SIR differs between synchronous and metachronous mRCC.

To the best of our knowledge, we reported for the first time that there were different prognostic roles of 2 SIR markers (PLR and NLR) between synchronous and metachronous mRCC patients receiving a first-line TKI. The prognostic significance of pretreatment PLR and NLR status was more significant in synchronous mRCC compared to metachronous mRCC. In addition, patients with increased NLR and PLR had a significantly poorer prognosis com-

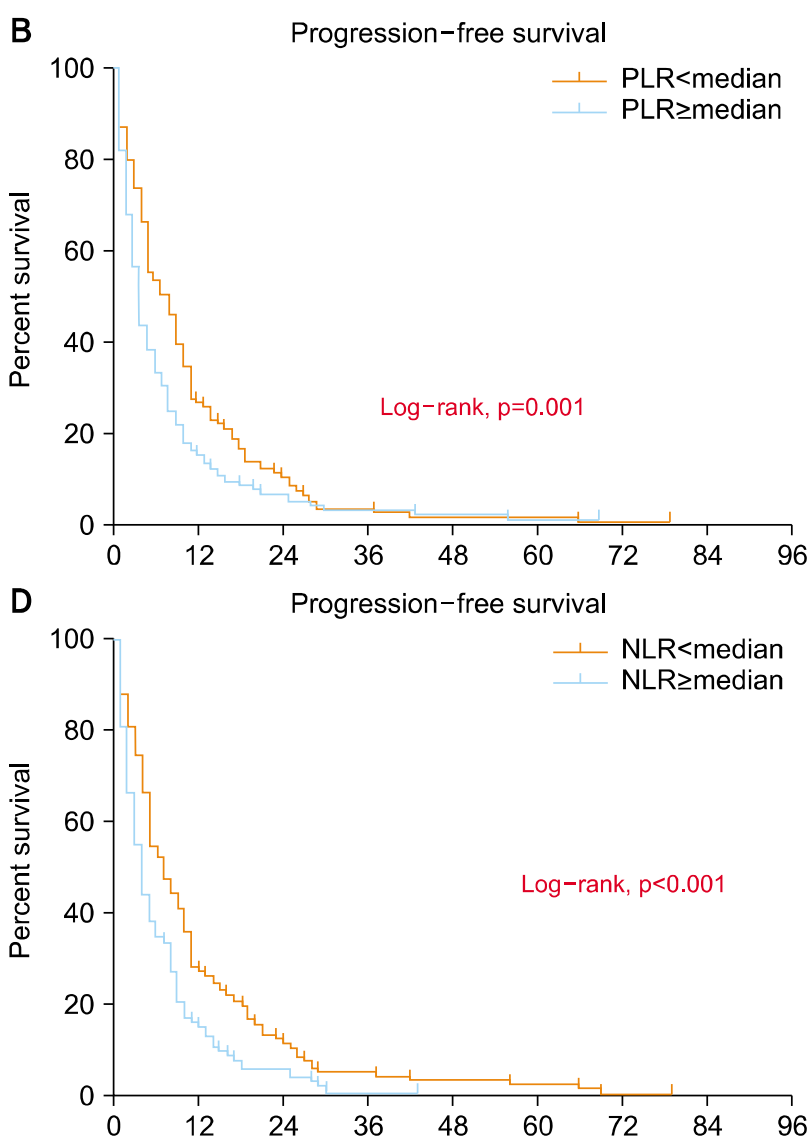

Fig. 2. Overall survival and progression-free survival in synchronous metastatic renal cell carcinoma patients stratified by median platelet-lymphocyte ratio (PLR) (A, B) and neutrophil-lymphocyte ratio (NLR) (C, D). 

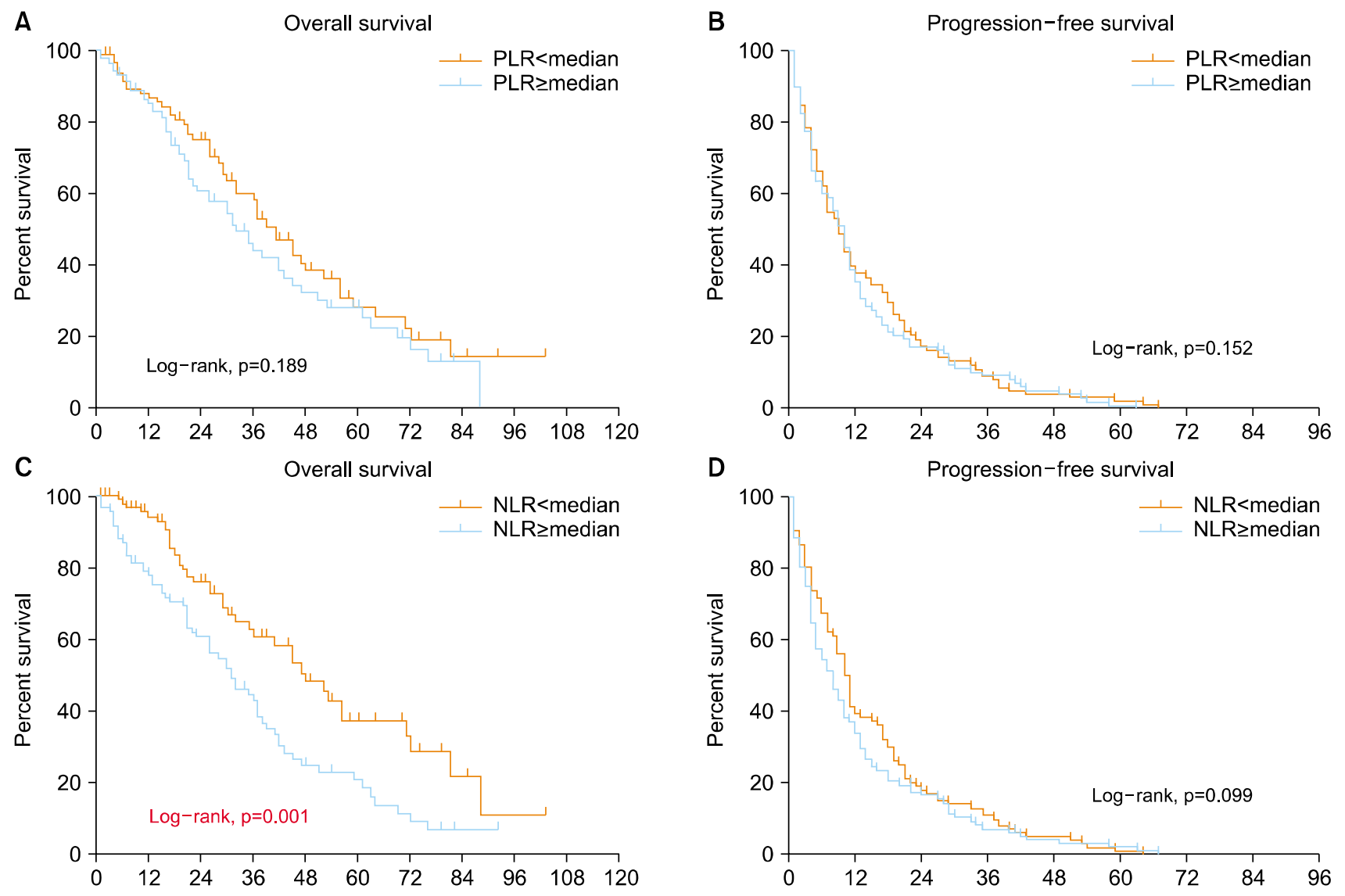

Fig. 3. Overall survival and progression-free survival in metachronous metastatic renal cell carcinoma patients stratified by medianplatelet-lymphocyte ratio (PLR) (A, B) and neutrophil-lymphocyte ratio (NLR) (C, D).

pared to those with lower NLR or PLR.

Recently, NLR and PLR which are markers of host inflammation have gained attention as prognostic markers in mRCC. ${ }^{18}$ There are relatively few reports on the relationship between PLR and RCC; however, it is well known that PLR is prognostic in several types of cancer such as large B-cell lymphoma, breast cancer, and colon cancer. ${ }^{19,20}$ In addition, reports indicate that NLR is related to metachronous metastasis of pancreatic cancer, lymph node metastasis of breast cancer, and peritoneal metastasis of gastric cancer. ${ }^{21,22}$

We found that NLR can predict OS outcomes regardless of synchronous or metachronous type $\mathrm{mRCC}$; however, the same was not true for PLR. Specifically, we found that PLR was not predictive of outcomes in metachronous mRCC patients. In other words, while NLR remained a significant independent predictor of OS, PLR failed to serve as a prognostic factor for OS and PFS in patients with metachronous mRCC. Templeton et al. ${ }^{18}$ showed that the proportion of immune cells in the blood is of prognostic value, and that ear- ly decline of NLR after targeted therapy is associated with favorable outcomes. Similarly, Zhang et al. ${ }^{23}$ showed that pretreatment NLR may be an independent prognostic factor in mRCC patients receiving targeted therapy. In our study, pretreatment lymphocytes were higher in metachronous mRCC. As a result of our literature search, there was no paper that reported relationship between metastasis or prognosis with lymphocytes alone. But many reports suggested that ratio with lymphocyte with various substances such as neutrophil, platelet, lactate dehydrogenase, or monocyte were cancer prognostic factor. ${ }^{24}$

One of the key findings of our study was that SIR markers were useful in synchronous $\mathrm{mRCC}$ patients but were of limited use in metachronous patients. Synchronous mRCC tumors are widespread in the short term and likely stimulate more interactions between the host and the tumor. ${ }^{25}$ In this way, there may be more opportunities for SIR markers to be meaningful, as the action of tumor infiltrating lymphocytes is expected to be more vigorous. 
Table 2. PLR - risk factor for OS with synchronous mRCC

\begin{tabular}{|c|c|c|c|c|}
\hline \multirow{2}{*}{ Variable } & \multicolumn{2}{|c|}{ Univariate } & \multicolumn{2}{|c|}{ Multivariate } \\
\hline & HR $(95 \%$ CI $)$ & p-value & HR $(95 \% \mathrm{CI})$ & $\mathrm{p}$-value \\
\hline \multicolumn{5}{|l|}{ Age (yr) } \\
\hline$<65$ & Reference & & & \\
\hline$\geq 65$ & $1.326(0.946-1.861)$ & 0.102 & & \\
\hline \multicolumn{5}{|l|}{ Sex } \\
\hline Male & Reference & & & \\
\hline Female & $1.114(0.765-1.622)$ & 0.573 & & \\
\hline \multicolumn{5}{|l|}{ BMI $\left(\mathrm{kg} / \mathrm{m}^{2}\right)$} \\
\hline$<25$ & Reference & & & \\
\hline$\geq 25$ & $0.549(0.361-0.836)$ & 0.005 & $0.610(0.385-0.967)$ & 0.036 \\
\hline \multicolumn{5}{|l|}{ IMDC criteria } \\
\hline Favorable & - & & - & \\
\hline Intermediate & Reference & & Reference & \\
\hline Poor & $1.967(1.402-2.759)$ & $<0.001$ & $1.668(1.150-2.418)$ & 0.007 \\
\hline \multicolumn{5}{|l|}{ Clinical $\mathrm{T}$ stage } \\
\hline$\leq \mathrm{cT} 2$ & Reference & & & \\
\hline$\geq \mathrm{cT} 3$ & $1.092(0.799-1.492)$ & 0.581 & & \\
\hline Cytoreductive nephrectomy & & $<0.001$ & & 0.007 \\
\hline None & Reference & & Reference & \\
\hline Radical & $0.456(0.334-0.623)$ & $<0.001$ & $0.570(0.399-0.816)$ & 0.002 \\
\hline Partial & $0.222(0.031-1.603)$ & 0.136 & $0.353(0.048-2.598)$ & 0.306 \\
\hline \multicolumn{5}{|l|}{ Multiple metastasis } \\
\hline Single & Reference & & & \\
\hline Multiple & $1.529(1.122-2.083)$ & 0.007 & & \\
\hline \multicolumn{5}{|l|}{ PLR } \\
\hline$<110.5$ & Reference & & Reference & \\
\hline$\geq 110.5$ & $1.985(1.470-2.679)$ & $<0.001$ & $1.556(1.112-2.179)$ & 0.010 \\
\hline
\end{tabular}

PLR: platelet-lymphocyte ratio, OS: overall survival, mRCC: metastatic renal cell carcinoma, HR: hazard ratio, CI: confidence interval, BMI: body mass index, IMDC: International Metastatic Renal Cell Carcinoma Database.

There is some evidence in the literature to suggest that synchronous and metachronous tumors have different characteristics. First, the prognosis of metachronous tumors are better than that of synchronous tumors. ${ }^{15}$ Second, Zheng et al. $^{26}$ showed different mutational patterns between synchronous and metachronous liver metastases of colorectal cancer. Third, a systematic review of colorectal cancer studies identified significant differences in expression of molecular markers between synchronous and metachronous metastases. ${ }^{27}$ We believe that the immune system plays a role in the differences between synchronous and metachronous disease. Unfortunately, in our search of the literature, we did not identify any reviews or studies focusing on the biological differences between synchronous and metachronous mRCC.

In this study, cytoreductive nephrectomy was an in- dependent predictor for OS and PFS in synchronous mRCC. These results are contrary to CARMENA trial, but CARMENA trial only contains MSKCC (Memorial Sloan Kettering Cancer Center) intermediate- and poor-risk patients. Also, unlike usual real-world settings, $43 \%$ were poor-risk patients in this study. ${ }^{28}$ Careful patient selection is more appropriate than complete abandon for cytoreductive nephrectomy. ${ }^{29}$

There were some limitations to this study. First, our study had a retrospective nature with potential of selection and classification bias. In addition, while the purpose of this study was to investigate the relationship between SIR and outcomes in synchronous and metachronous mRCC, it is difficult to make firm conclusions from a single blood test measurement prior to starting TKI treatment. Third, in some cases of metachronous RCC patients, the results of previous 
Table 3. PLR - risk factor for OS with metachronous $\mathrm{mRCC}$

\begin{tabular}{|c|c|c|c|c|}
\hline \multirow{2}{*}{ Variable } & \multicolumn{2}{|c|}{ Univariate } & \multicolumn{2}{|c|}{ Multivariate } \\
\hline & HR $(95 \%$ CI $)$ & p-value & HR $(95 \% \mathrm{CI})$ & $\mathrm{p}$-value \\
\hline \multicolumn{5}{|l|}{ Age (yr) } \\
\hline$<65$ & Reference & & & \\
\hline$\geq 65$ & $1.050(0.698-1.577)$ & 0.816 & & \\
\hline \multicolumn{5}{|l|}{ Sex } \\
\hline Male & Reference & & & \\
\hline Female & $0.741(0.440-1.250)$ & 0.262 & & \\
\hline \multicolumn{5}{|l|}{ BMI $\left(\mathrm{kg} / \mathrm{m}^{2}\right)$} \\
\hline$<25$ & Reference & & & \\
\hline$\geq 25$ & $0.848(0.521-1.381)$ & 0.508 & & \\
\hline IMDC criteria & & 0.312 & & 0.034 \\
\hline Favorable & Reference & & Reference & \\
\hline Intermediate & $1.234(0.830-1.833)$ & 0.299 & $1.725(1.087-2.738)$ & 0.021 \\
\hline Poor & $2.183(0.669-7.115)$ & 0.195 & $2.760(0.829-9.197)$ & 0.098 \\
\hline \multicolumn{5}{|l|}{ Clinical $\mathrm{T}$ stage } \\
\hline$\leq \mathrm{cT} 2$ & Reference & & & \\
\hline$\geq \mathrm{cT} 3$ & $1.334(0.881-2.020)$ & 0.174 & & \\
\hline Grade & & 0.334 & & \\
\hline 1 & Reference & & & \\
\hline 2 & $1.426(0.875-2.324)$ & 0.155 & & \\
\hline 3 & $1.159(0.569-2.361)$ & 0.685 & & \\
\hline \multicolumn{5}{|l|}{ Histology } \\
\hline Clear cell & Reference & & & \\
\hline Nonclear cell & $1.208(0.583-2.501)$ & 0.611 & & \\
\hline \multicolumn{5}{|c|}{ Multiple metastasis } \\
\hline Single & Reference & & Reference & \\
\hline Multiple & $1.527(1.026-2.272)$ & 0.037 & $1.470(0.939-2.300)$ & 0.092 \\
\hline \multicolumn{5}{|l|}{ PLR } \\
\hline$<90.4$ & Reference & & & \\
\hline$\geq 90.4$ & $1.283(0.872-1.886)$ & 0.206 & & \\
\hline
\end{tabular}

PLR: platelet-lymphocyte ratio, OS: overall survival, mRCC: metastatic renal cell carcinoma, HR: hazard ratio, CI: confidence interval, BMI: body mass index, IMDC: International Metastatic Renal Cell Carcinoma Database.

treatment and biopsy results were unclear. Thus, due to this limitation, we conducted the risk analysis of cytoreductive nephrectomy in only synchronous RCC patient. Finally, we only tested blood samples to evaluate PLR and NLR as markers of SIR, rather than concurrently assess for tumor inflating cells in pathologic slides. Despite these drawbacks, our study describes for the first time the different prognostic power of the SIR markers PLR and NLR between synchronous and metachronous mRCC. The importance of SIR is expected to increase in the era of immune-oncology, and it can be useful to apply SIR markers according to synchronous and metachronous $\mathrm{mRCC}$ disease in appropriate clinical settings.

\section{CONCLUSIONS}

In summary, we found that SIR such as PLR and NLR have different prognostic value in synchronous and metachronous mRCC patients who were treated with first-line TKI. In particular, SIRs were more useful in synchronous mRCC population. Our study provides valuable information regarding the ability to predict survival outcomes in different types of mRCC by using well-known SIR markers.

\section{CONFLICT OF INTEREST}

The authors claim no conflicts of interest. 


\section{SUPPLEMENTARY MATERIALS}

Supplementary Tables 1-6 can be found via https://do i.org/10.22465/kjuo.2019.17.3.150.

\section{REFERENCES}

1. Motzer RJ, Jonasch E, Agarwal N, Beard C, Bhayani S, Bolger GB, et al. Kidney cancer, version 3.2015. J Natl Compr Canc Netw 2015;13:151-9.

2. Motzer RJ, Escudier B, McDermott DF, George S, Hammers HJ, Srinivas S, et al. Nivolumab versus Everolimus in Advanced Renal-Cell Carcinoma. N Engl J Med 2015;373:1803-13.

3. Motzer RJ, Tannir NM, McDermott DF, Arén Frontera O, Melichar B, Choueiri TK, et al. Nivolumab plus Ipilimumab versus Sunitinib in Advanced Renal-Cell Carcinoma. N Engl J Med 2018;378:1277-90.

4. Subramanian P, Haas NB. Recent advances in localized RCC: a focus on VEGF and immuno-oncology therapies. Urol Oncol 2018;36:23-30.

5. Mataraza JM, Gotwals P. Recent advances in immuno-oncology and its application to urological cancers. BJU Int 2016;118:506-14.

6. Zhang LX, Wei ZJ, Xu AM, Zang JH. Can the neutrophil-lymphocyte ratio and platelet-lymphocyte ratio be beneficial in predicting lymph node metastasis and promising prognostic markers of gastric cancer patients? Tumor maker retrospective study. Int J Surg 2018;56:320-7.

7. Wang W, Liu W, Zhang N, He X. Preoperative platelet-lymphocyte ratio is an independent prognostic factor in ampullary carcinoma following pancreaticoduodenectomy. Oncol Lett 2018;16:4879-88.

8. Temur I, Kucukgoz Gulec U, Paydas S, Guzel AB, Sucu M, Vardar MA. Prognostic value of pre-operative neutrophil/lymphocyte ratio, monocyte count, mean platelet volume, and platelet/lymphocyte ratio in endometrial cancer. Eur J Obstet Gynecol Reprod Biol 2018;226:25-9.

9. Vartolomei MD, Porav-Hodade D, Ferro M, Mathieu R, Abufaraj M, Foerster B, et al. Prognostic role of pretreatment neutrophil-to-lymphocyte ratio (NLR) in patients with non-muscle-invasive bladder cancer (NMIBC): A systematic review and meta-analysis. Urol Oncol 2018;36: 389-99.

10. Rajwa P, Życzkowski M, Paradysz A, Bujak K, Bryniarski P. Evaluation of the prognostic value of LMR, PLR, NLR, and dNLR in urothelial bladder cancer patients treated with radical cystectomy. Eur Rev Med Pharmacol Sci 2018;22:3027-37.

11. Bilen MA, Dutcher GMA, Liu Y, Ravindranathan D,
Kissick HT, Carthon BC, et al. Association Between Pretreatment Neutrophil-to-Lymphocyte Ratio and Outcome of Patients With Metastatic Renal-Cell Carcinoma Treated With Nivolumab. Clin Genitourin Cancer 2018;16:e56375 .

12. Tanaka N, Mizuno R, Yasumizu Y, Ito K, Shirotake S, Masunaga A, et al. Prognostic value of neutrophil-to-lymphocyte ratio in patients with metastatic renal cell carcinoma treated with first-line and subsequent second-line targeted therapy: a proposal of the modified-IMDC risk model. Urol Oncol 2017;35:39.e19-39.e28.

13. Wang Z, Peng S, Wang A, Xie H, Guo L, Jiang N, et al. Platelet-lymphocyte ratio acts as an independent predictor of prognosis in patients with renal cell carcinoma. Clin Chim Acta 2018;480:166-72.

14. Chrom P, Stec R, Bodnar L, Szczylik C. Incorporating Neutrophil-to-lymphocyte Ratio and Platelet-to-lymphocyte Ratio in Place of Neutrophil Count and Platelet Count Improves Prognostic Accuracy of the International Metastatic Renal Cell Carcinoma Database Consortium Model. Cancer Res Treat 2018;50:103-10.

15. Kim SH, Suh YS, Lee DE, Park B, Joo J, Joung JY, et al. A retrospective comparative study of progression-free survival and overall survival between metachronous and synchronous metastatic renal cell carcinoma in intermediate- or poor-risk patients treated with VEGF-targeted therapy. Oncotarget 2017;8:93633-43.

16. Szendi B, Lakatos I. Synchronous \& metachronous malignant multiple tumors of the female genitalia. Oncologia 1959;12:49-60.

17. Gutenberg A, Nischwitz MD, Gunawan B, Enders C, Jung $\mathrm{K}$, Bergmann $\mathrm{M}$, et al. Predictive chromosomal clusters of synchronous and metachronous brain metastases in clear cell renal cell carcinoma. Cancer Genet 2014;207:206-13.

18. Templeton AJ, Knox JJ, Lin X, Simantov R, Xie W, Lawrence N, et al. Change in Neutrophil-to-lymphocyte Ratio in Response to Targeted Therapy for Metastatic Renal Cell Carcinoma as a Prognosticator and Biomarker of Efficacy. Eur Urol 2016;70:358-64.

19. Zhu Y, Si W, Sun Q, Qin B, Zhao W, Yang J. Plateletlymphocyte ratio acts as an indicator of poor prognosis in patients with breast cancer. Oncotarget 2017;8:1023-30.

20. Yang L, He W, Kong P, Jiang C, Yang Q, Xie Q, et al. Clinical baseline and prognostic difference of platelet lymphocyte ratio (PLR) in right-sided and let-sided colon cancers. BMC Cancer 2017;17:873.

21. Arima K, Okabe H, Hashimoto D, Chikamoto A, Nitta H, Higashi T, et al. Neutrophil-to-lymphocyte ratio predicts metachronous liver metastasis of pancreatic neuroendocrine tumors. Int J Clin Oncol 2017;22:734-9.

22. Eroglu A, Akbulut S. The Role of Pretreatment Neutrophil to Lymphocyte Ratio and Platelet to Lymphocyte Ratio in 
the Diagnosis of Breast Cancer: Predicting Lymph Node Metastasis. World J Oncol 2013;4:262-3.

23. Zhang GM, Zhu Y, Gu WJ, Zhang HL, Shi GH, Ye DW. Pretreatment neutrophil-to-lymphocyte ratio predicts prognosis in patients with metastatic renal cell carcinoma receiving targeted therapy. Int J Clin Oncol 2016;21:373-8.

24. Li T, Li H, Xie S, Tan Y, Xie ZP, Li WY, et al. Lactate Dehydrogenase-to-Lymphocyte Ratio Represents a Powerful Prognostic Tool of Metastatic Renal Cell Carcinoma Patients Treated with Tyrosine Kinase Inhibitors. Pathol Oncol Res 2019 Aug 6. doi: 10.1007/s12253-019-00707-z. [Epub ahead of print]

25. Pogge von Strandmann E, Reinartz S, Wager U, Müller R. Tumor-Host Cell Interactions in Ovarian Cancer: Pathways to Therapy Failure. Trends Cancer 2017;3:13748.

26. Zheng P, Ren L, Feng Q, Zhu D, Chang W, He G, et al.
Differences in clinical characteristics and mutational pattern between synchronous and metachronous colorectal liver metastases. Cancer Manag Res 2018;10:2871-81.

27. Slesser AA, Georgiou P, Brown G, Mudan S, Goldin R, Tekkis $\mathrm{P}$. The tumour biology of synchronous and metachronous colorectal liver metastases: a systematic review. Clin Exp Metastasis 2013;30:457-70.

28. Mejean A, Ravaud A, Thezenas S, Colas S, Beauval JB, Bensalah K, et al. Sunitinib Alone or after nephrectomy in metastatic renal-cell carcinoma. N Engl J Med. 2018; 379:417-27

29. Bhindi B, Abel EJ, Albiges L, Bensalah K, Boorjian SA, Daneshmand S, et al. Systematic Review of the Role of Cytoreductive Nephrectomy in the Targeted Therapy Era and Beyond: An Individualized Approach to Metastatic Renal Cell Carcinoma. Eur Urol 2019;75:111-28. 